LBL-37084

UC-414

\title{
Utilization of Amorphous Silicon Carbide (a-Si:C:H) as a Resistive Layer in Gas Microstrip Detectors
}

\author{
W.-S. Hong, H..-S. Cho, V. Perez-Mendez, and W.G. Gong* \\ Physics Division and *Nuclear Science Division \\ Lawrence Berkeley Laboratory \\ University of California \\ Berkeley, California 94720
}

\section{DISCLAIMER}

\begin{abstract}
This report was prepared as an account of work sponsored by an agency of the United States Government. Neither the United States Government nor any agency thereof, nor any of their employees, makes any warranty, express or implied, or assumes any legal liability or responsibility for the accuracy, completeness, or usefulness of any information, apparatus, product, or process disclosed, or represents that its use would not infringe privately owned rights. Reference herein to any specific commercial product, process, or service by trade name, trademark, manufacturer, or otherwise does not necessarily constitute or imply its endorsement, recommendation, or favoring by the United States Government or any agency thereof. The views and opinions of authors expressed herein do not necessarily state or reflect those of the United States Government or any agency thereof.
\end{abstract}

This work was supported by the Director, Office of Energy Research, Office of High Energy and Nuclear Physics, Division of High Energy Physics, of the U.S. Department of Energy under Contract No. DE-ACO3$76 \mathrm{SF} 00098$. 


\section{DISCLAIMER}

Portions of this document may be illegible in electronic image products. Images are produced from the best available original document. 


\title{
UTILIZATION OF AMORPHOUS SILICON CARBIDE (a-Si:C:H) AS A RESISTIVE LAYER IN GAS MICROSTRIP DETECTORS
}

\author{
W.S. HONG, H.S. CHO, V. PEREZ-MENDEZ and W.G.GONG* \\ Physics Division, *Nuclear Science Division, Lawrence Berkeley Laboratory, Berkeley, CA \\ 94720
}

\begin{abstract}
Thin semiconducting films of hydrogenated amorphous silicon (a-Si:H) and its carbon alloy (a-Si:C:H) were applied to gas microstrip detectors in order to control gain instabilities due to charges on the substrate. Thin $(\sim 100 \mathrm{~nm})$ layers of a-Si:H or p-doped a-Si:C:H were placed either over or under the electrodes using the plasma enhanced chemical vapor deposition (PECVD) technique to provide the substrate with a suitable surface conductivity. By changing the carbon content and boron doping density, the sheet resistance of the a-Si:C:H coating could be successfully controlled in the range of $10^{12} \sim 10^{17} \Omega / \square$, and the light sensitivity, which causes the resistivity to vary with ambient light conditions, was minimized. An avalanche gain of 5000 and energy resolution of $20 \%$ FWHM were achieved and the gain remained constant over a week of operation. A-Si: $\mathrm{C}: \mathrm{H}$ film is an attractive alternative to ion-implanted or semiconducting glass due to the wide range of resistivities possible and the feasibility of making deposits over a large area at low cost.
\end{abstract}

\section{INTRODUCTION}

Gas Microstrip Detector (GMD) is a miniatured version of the multiwire proportional counter with improved spatial resolution, energy resolution and rate capability. The wires are replaced by metallic strips printed on the surface of insulators. Incident radiation ionizes the gas between the drift plane and the microstrip plane and the electrons are multiplied by the gas avalanche process in the intense electric field.

However, the long term instability and rate-dependent instability of gas gain due to charging on the surface of the insulator impose limitation on the use of the microstrip structure. Fig. 1. shows the cross-section of a GMD. The incident radiation ionizes the gas molecule and the resulting electrons are accelerated toward the anode. Since the mobility of positive ions are much smaller than that of electrons, the ions tend to pile up near the anode. The accumulation of the 


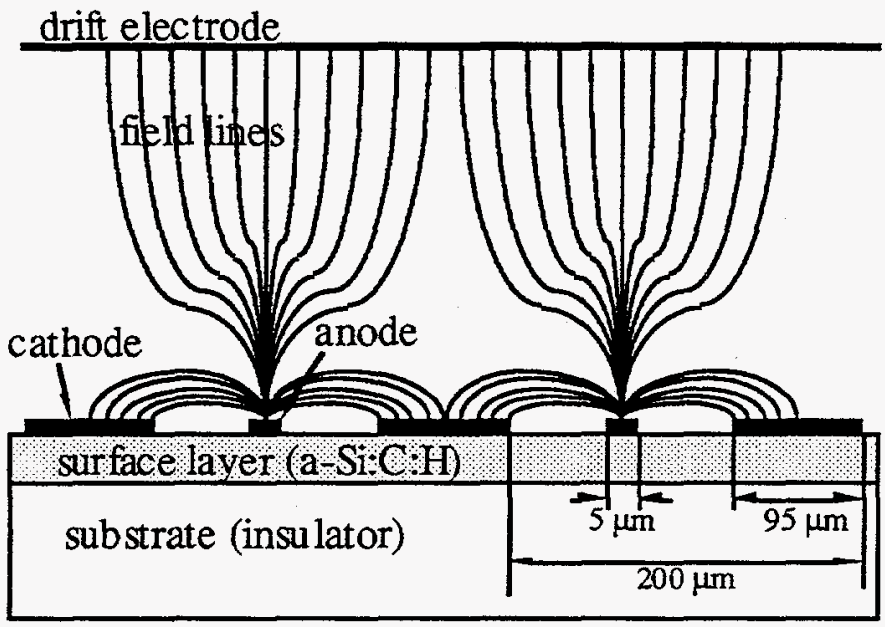

Fig. 1 Schematic diagram of the cross section of a gas microstrip detector . positive charges modifies the electric field profile and decreases the avalanche gain. This charging-up effect can be reduced by introducing an additional electrode, whose potential is somewhere between those of the drift electrode and the anode, between the two adjacent anodes. The ions accumulated around the anode are swept out towards this intermediate electrode, namely cathode, through the potential lines drawn in Fig. 1. Positive ions landing on the surface of the substrate do not move if the substrate is insulating.

The substrate thus requires a certain conductivity to neutralize the positive charges depositing on the surface. At the same time, the surface conductivity should not be too high, because then the leakage current between the anode and cathode is large enough to produce excessive noise above the signal level. It has been shown empirically that the surface resistivity must be within the range of $10^{12} \sim 10^{16} \Omega / \square$ to provide a stable avalanche gain.[1] Therefore, finding a suitable substrate material with resistivity in the above range has been of primary interest. Among the strategies to achieve the desired resistivity are ion-implantation of insulating materials and sputter-coating of thin semiconducting films.[2-4] Both good long-term stability and high rate capability have been obtained by these methods. However, ion-implantation is much more expensive than ordinary thin-film coating process. Moreover, charging-up of electrons at the insulating substrate during implantation makes it difficult to control the uniformity and dosage accurately. Sputtering of conducting material is relatively easy and cheap, but it is hard to change the resistivity in a broad range since the resistivity is controlled mostly by varying the thickness.

\section{APPLICATION OF a-Si:H TO SURFACE COATING}

Hydrogenated amorphous silicon (a-Si:H) and its alloys are good candidates for a semiconducting substrate for gas microstrip detectors (GMDs) due to their good radiation hardness and the feasibility of depositing over a wide area at low cost. Not only is the bulk resistivity of a-Si:H layer in a suitable range for a $\mathrm{GMD}\left(\sim 10^{9} \Omega \cdot \mathrm{cm}\right)$, it can also be easily controlled over a wide range by doping or alloying in the gas phase. However, as reported by Savard et al,,[5] intrinsic a-Si:H 
layers showed variation in surface resistivity and gain with the backplane voltages $\left(\mathrm{V}_{\mathrm{b}}\right)$. They attributed such a behavior to the undepleted carriers in a-Si:H, i.e., non-bonding valence electrons (dangling bonds). We also observed that it takes several hours to reach the equilibrium value of the surface resistivity. Another feature of a-Si:H film is its light sensitivity. The resistivity decreases by 3 or 4 orders of magnitude when exposed to ambient light. Variation of the surface resistivity will change the leakage current between anodes and cathode, which in turn introduces uncertainty to the gas gain. Even when tightly sealed from its surrounding, the UV light generated during the avalanche process may affect the gain.

Therefore, we attempted to minimize light sensitivity and the effect of the dangling bonds to improve the performance of the a-Si:H based detectors. One way is to make the electronic conduction dominated by extrinsic carriers, i.e., dopants. Since the sheet resistance falls below the suitable resistivity range $\left(10^{12}-10^{16} \Omega / \square\right)$ when the number of extrinsic carriers exceeds that of intrinsic carriers (ionizable dangling bonds), it must be compensated by alloying with other elements. One of the best candidates is carbon since it is in the same column in the periodic table as $\mathrm{Si}$ and addition of $\mathrm{C}$ to $\mathrm{Si}$ is known to increase the bandgap and resistivity. In this paper, we present test results of gas microstrip detectors having doped a-Si:C:H coatings on surface, and compare them with the results obtained from detectors made on insulating glass surface and on other semiconducting materials.

\section{EXPERIMENTAL PROCEDURES}

An alternating anode and cathode pattern was formed with a $0.2 \mu \mathrm{m}$-thick $\mathrm{Cr}$ layer on a 0.8 mm-thick Corning 7059 glass substrate using the sputtering and lift-off method to ensure a uniform edge profile. The anode and the cathode widths were $5 \mu \mathrm{m}$ and $95 \mu \mathrm{m}$, respectively, and the anode to anode pitch was $200 \mu \mathrm{m}$. The active area of the detector was $15 \mathrm{~mm} \times 20 \mathrm{~mm}$. A layer of a-Si:C:H approximately $100 \mathrm{~nm}$-thick were deposited by the plasma enhanced chemical vapor deposition (PECVD) technique either before or after the electrodes were made. The carbon content and doping density in the a-Si:C:H films were controlled by changing the relative flow rates of the $\mathrm{CH}_{4}$ and $\mathrm{B}_{2} \mathrm{H}_{6}$. Ten anode strips were grouped together and biased with a positive voltage. All cathode strips were interconnected and held at a ground potential. The dark surface resistivity was calculated from the sample geometry and leakage current between anode and cathode. The photoconductivity was measured under $60 \mathrm{~cd}-\mathrm{ft}$. white light illumination.

The microstrip plate was mounted on a printed circuit board inside an aluminum chamber with an $\mathrm{O}$-ring seal. The drift plane was made of a thin aluminum wire grid and was located $4 \mathrm{~mm}$ above the microstrip plate. $1: 1 \mathrm{Ar}-\mathrm{CH}_{4}$ or $\mathrm{Ar}-\mathrm{C}_{2} \mathrm{H}_{6}$ mixture was used as fill gas with a flow rate 


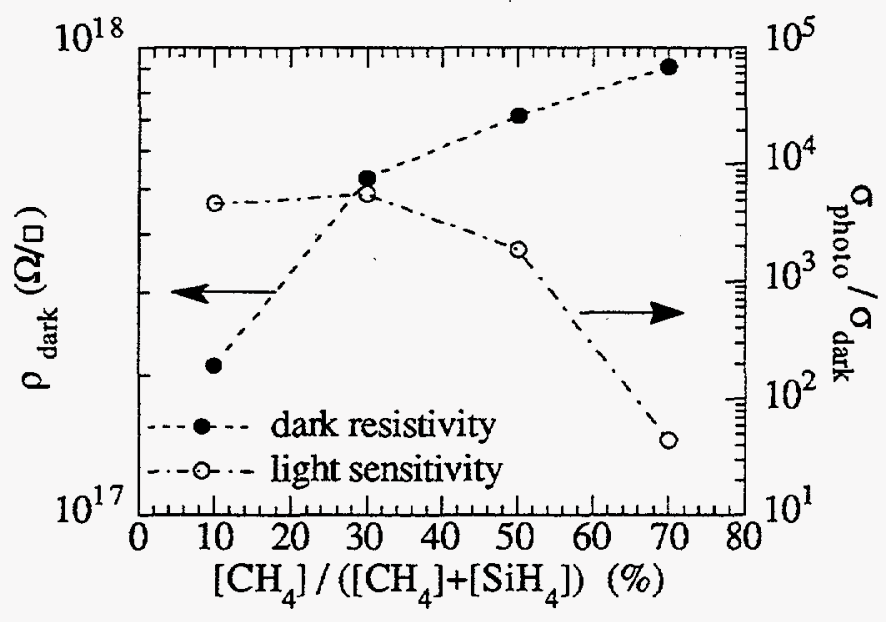

Fig. 2 Variations of dark surface resistivity and light sensitivity as a function of $\mathrm{CH}_{4}$ content in the source gas. of $100 \mathrm{sccm}$. The anode readout is capacitively coupled to a chargesensitive preamplifier. The signal charges were then integrated by a shaping amplifier and processed by a pulse height analyzer. The detectors were tested with a ${ }^{55} \mathrm{Fe}$ source which emits $5.9 \mathrm{keV} \mathrm{X}$-ray. Gas avalanche gain, energy resolution, timedependent stability and ageing under prolonged irradiation were studied from the recorded pulse height spectrum.

\section{RESULTS AND DISCUSSION}

The dark surface resistivity and the light sensitivity as functions of the $\mathrm{CH}_{4}$ content in the process gas are plotted in Fig. 2. The light sensitivity is defined as the ratio of photoconductivity to dark conductivity. The dark resistivity increased and the light sensitivity decreased with the $\mathrm{CH}_{4}$ content. The dark resistivity increased rapidly up to a $\mathrm{CH}_{4}$ content of $30 \%$ and then slowed down. The increase in the dark resistivity with increasing carbon content is due to the combined effect of reduced carrier mobility and the number of carriers participating in electrical transport. The electron drift mobility of a-Si:C:H films having band gap energies of $1.75 \sim 1.9 \mathrm{eV}$ are reported to be 1 3 orders of magnitude lower than that of pure a-Si:H.[6] Therefore, the initial abrupt increase in resistivity with carbon content is believed to be mainly due to the decrease in electron mobility.

The light sensitivity did not change appreciably until the $\mathrm{CH}_{4}$ content is increased to $50 \%$. According to other works, the optical gap of the material with a $50 / 50 \mathrm{SiH}_{4}-\mathrm{CH}_{4}$ mixture is between $1.9 \sim 2.3 \mathrm{eV}$. The maximum value of the optical band gap energy is about $2.7 \mathrm{eV}$ when the atomic fraction of carbon incorporated in the film $\mathrm{x}=0.65 .[7,8]$ This gap energy corresponds to the wavelength of $460 \mathrm{~nm}$ (blue light); thus alloying with carbon solely cannot effectively prevent conductivity change due to incident photons when illuminated with white light. Moreover, the undoped a-Si:C:H is transparent to UV. 


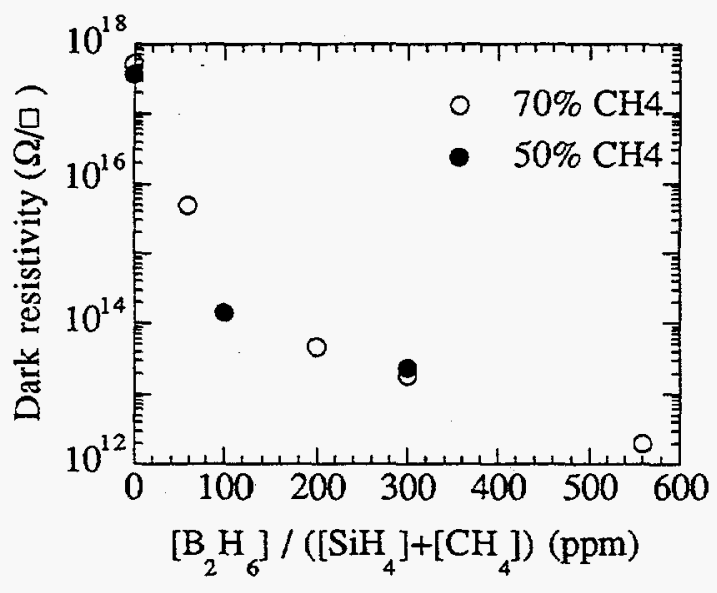

(a)

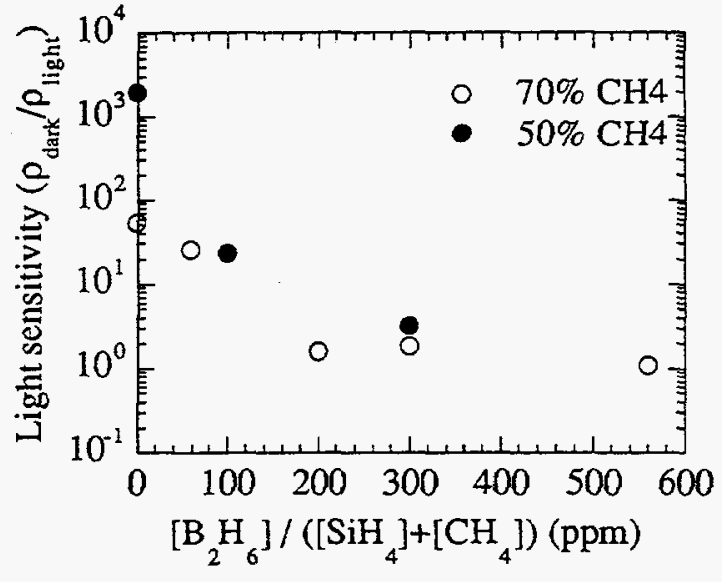

(b)

Fig. 3. Change in (a) dark resistivity and (b) light sensitivity with gas phase boron doping density

In order to suppress the light sensitivity further, gas phase doping was performed. If a semiconductor is heavily doped, the conductivity will be determined by the doping level and not be affected significantly by temperature or illumination conditions. Statistically, if the number of extrinsic carriers introduced by doping exceeds that of the intrinsic carriers, the overall conductivity will not change significantly by a small variation of the number of electron-hole pairs produced by light. Fig. 3 shows variation in dark resistivity and light sensitivity with the boron doping concentration for samples prepared from gas mixtures containing $50 \%$ and $70 \% \mathrm{CH}_{4}$, respectively. It is clearly seen for both sets of data that the light sensitivity decreases with doping density. With a gas phase doping density of $600 \mathrm{ppm}$, a-Si:C:H deposited from a 30/70 $\mathrm{SiH}_{4}-\mathrm{CH}_{4}$ mixture did not show any variation in resistivity at the given illumination conditions.

The time dependent leakage current behavior is characterized by an initial sharp increase upon application of the bias voltage followed by a gradual decay with time. In pure a- $\mathrm{Si}: \mathrm{H}$, it took several hours to reach a steady-state value of the leakage current. Doping shortens the time to reach the equilibrium leakage current between the anode and cathode. Time dependence of the surface resistivity calculated from the leakage current is plotted in Fig. 4 for devices with undoped a-Si:H layer and p-doped a-Si:C:H layers. The surface resistivity values were normalized to the steady-state value. The p-doped a-Si: $\mathrm{C}: \mathrm{H}$ reached equilibrium within 10 minutes. The origin of the time-dependence of the leakage current is not well understood. However, the slow equilibration in an undoped a-Si:H sample seems to be due the release of charge carriers from the dangling bonds under a strong electric field.[9] The dangling bonds occupy energy levels deep down in the band gap ( $0.9 \mathrm{eV}$ from the conduction band edge), whereas the acceptor dopant level 


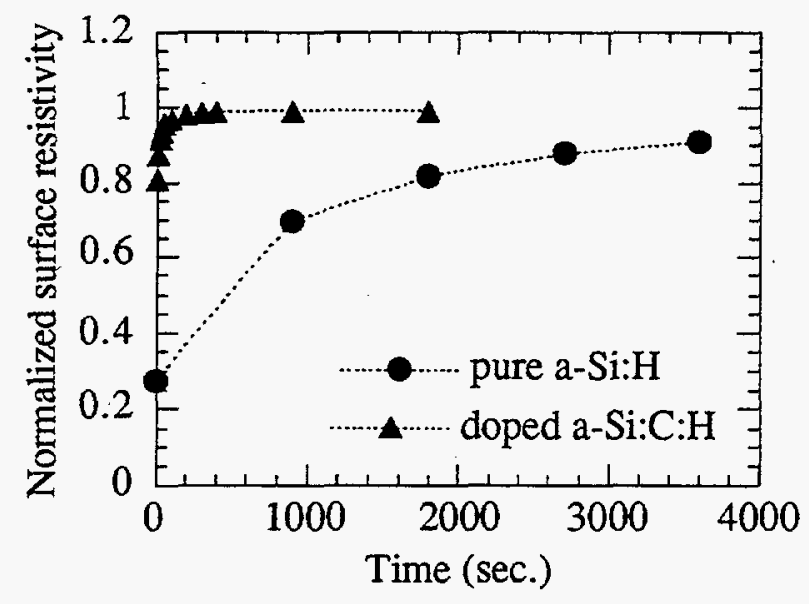

Fig. 4. Time dependent surface resistivity normalized to a steady-state value.

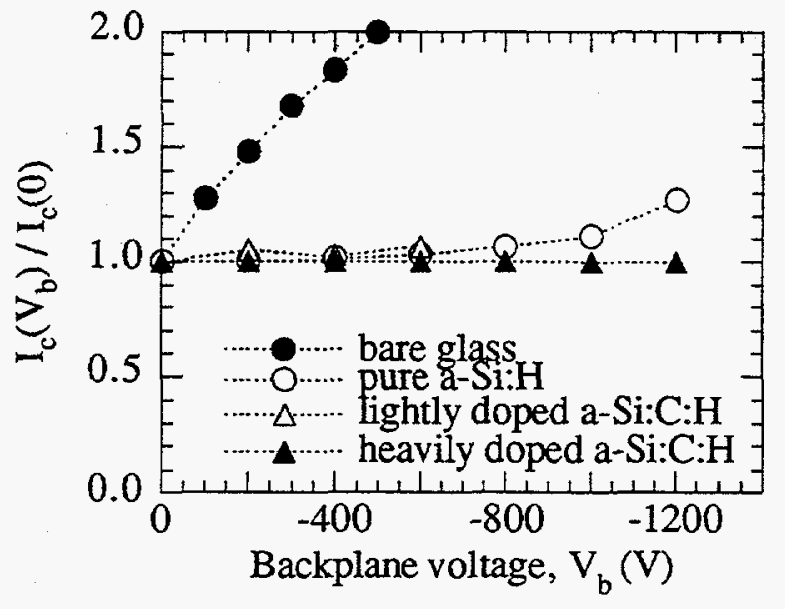

Fig. 5. Back-plane voltage dependence of the normalized cathode current. is only $\sim 0.2 \mathrm{eV}$ above the valence band edge. The relaxation time of the trapped carriers, which is defined as the time at which the current is 1.1 times its steadystate value, increases exponentially with the position of the energy levels of the deep traps relative to either band edge.[10] Therefore, it takes more time to reach a steady-state value in the undoped a-Si:H than in the doped a-Si:C:H where the number of extrinsic carriers introduced by doping is much greater than the charge carriers released from the dangling bonds.

When tested with a $5.9 \mathrm{keV} \mathrm{X}$-ray from an ${ }^{55} \mathrm{Fe}$ source, a maximum gas gain of $\sim 5000$, rate capabilities of $\sim 10^{5}$ counts $/ \mathrm{mm}^{2} \cdot \mathrm{sec}$, and energy resolution of $\leq 20 \%$ FWHM were achieved. In all samples, the gas gain increased exponentially with the anode bias until the voltage tripped out. Both devices with the a-Si:C:H layer under and over the electrodes worked well, but as reported by Bouclier et al.[11], coating the semiconducting layer over the electrodes greatly simplified the fabrication procedure. In both cases, the gain did not change much either with time or with $V_{b}$.

Change in cathode current with backplane voltage $\mathrm{V}_{\mathrm{b}}$ is plotted in Fig.5. The sample without surface coating showed a noticeable dependence of $\mathrm{V}_{b}$ as reported by others.[12] The pure a-Si:H layer showed a slight dependence on $\mathrm{V}_{b}$, but the variation is not as significant as reported by Savard et al.[5] This is probably because our insulating surface is 470 times thicker than theirs and the electric field seen by the surface layer is relatively small. The lightly doped sample $\left(60 \mathrm{ppm} \mathrm{B}_{2} \mathrm{H}_{6}\right)$ still had some dependence in the cathode current with $\mathrm{V}_{\mathbf{b}}$. The heavily doped sample ( $\left.600 \mathrm{ppm} \mathrm{B}_{2} \mathrm{H}_{6}\right)$ showed no noticeable current variation up to the backplane 


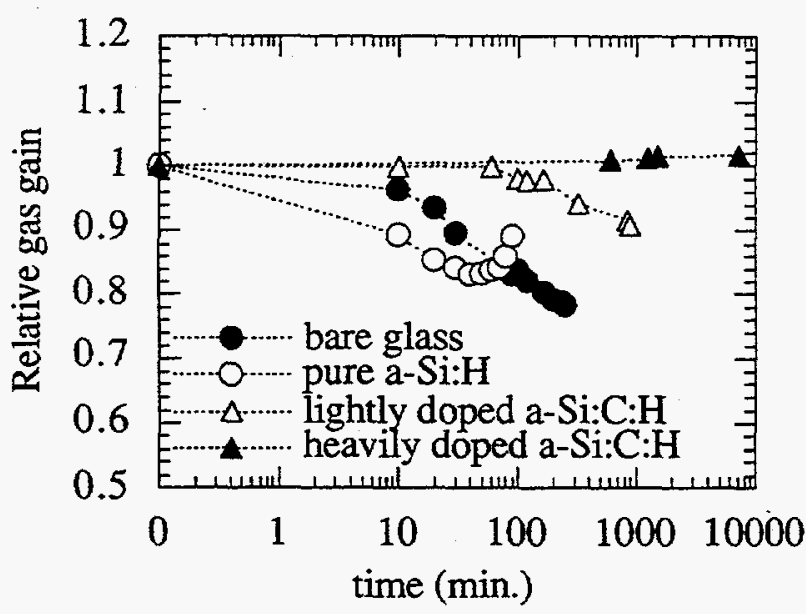

Fig.6 Time dependence of the relative gas gain.

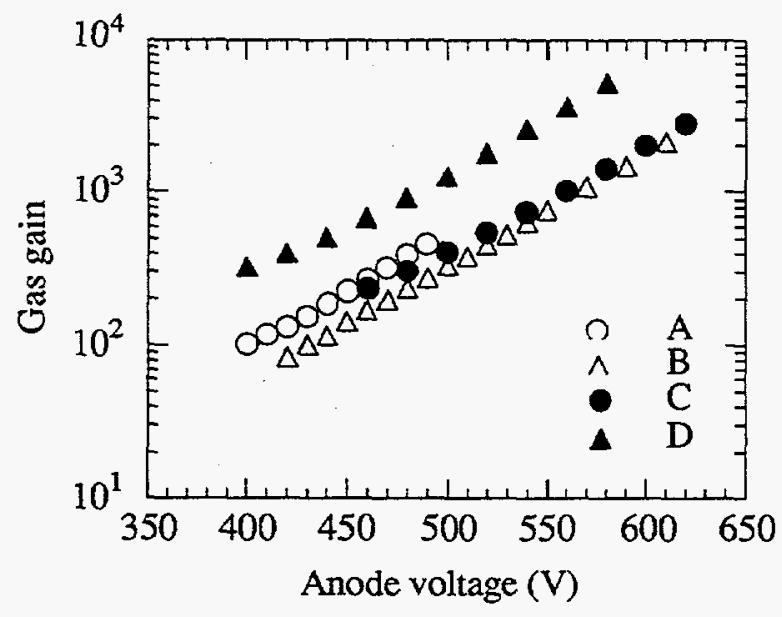

Fig. 7. Gas gains of GMDs having two types of surface layers in various gas mixtures. Sample configurations and test conditions are listed in Table I.

voltages as high as $-1200 \mathrm{~V}$, indicating that this layer effectively screens the electric field induced by the backplane voltage.

In Fig. 6, changes in normalized gas gain with time are plotted for four different types of detectors. For the sample having no coating on the surface, a gain drop of $\sim 30 \%$ was observed within the first 10 minutes, because the surface resistivity $\left(10^{17} \Omega / \Omega\right)$ is higher than the optimum range. For the detector with pure a-Si:H coating, the gain decreased first and then increased slightly as time passed. For heavily p-doped a-Si:C:H, the gain remained constant over a period of a week, indicating that the coated layer effectively prevents the positive ions from accumulating on the detector surface.

For comparison, we also tested GMDs made on substrates coated with $0.5 \sim 1.0 \mu \mathrm{m}$ thick $\mathrm{S} 8900$ glass layers, which have electronic conductivity. The results are shown in Fig. 7. Sample configurations and test conditions are summarized in Table $I$. Performance of the detectors with a-Si:C:H layer was comparable to that with electronicconductive glass coating. The sheet resistance was $\sim 10^{12} \Omega / \square$ in both devices. The samples showed similar gain behavior irrespective of the coating material and fill gas. Only the increase of the cathode to anode width ratio from 20 to 40 improved the gas gain by a factor of $1.5 \sim 2$ at the expense of the anode-to-anode pitch. Clearly, doped a-Si:C:H films are a good alternative to semiconducting glass.

Ageing is another problem in gaseous devices which limits high rate capability. Permanent damage to the electrodes after extended irradiation and a severe degradation of performance have been associated with the production in the avalanches of polymeric compounds that stick to anodes 
Table I. Sample configurations and test conditions of various detectors in Fig. 7.

\begin{tabular}{cccccc}
\hline Sample & Surface layer & Pitch & $\begin{array}{c}\text { Cathode-to-anode } \\
\text { width ratio }\end{array}$ & Fill gas & $\begin{array}{c}\text { Drift } \\
\text { voltage }\end{array}$ \\
\hline A & S8900 glass & $200 \mu \mathrm{m}$ & 9 & $\mathrm{Ar}-\mathrm{CH}_{4}$ & $-200 \mathrm{~V}$ \\
B & S8900 glass & $200 \mu \mathrm{m}$ & 9 & $\mathrm{He}-\mathrm{C}_{2} \mathrm{H}_{6}$ & $-600 \mathrm{~V}$ \\
C & p-doped a-Si:C:H & $200 \mu \mathrm{m}$ & 20 & $\mathrm{Ar}-\mathrm{C}_{2} \mathrm{H}_{6}$ & $-1000 \mathrm{~V}$ \\
D & p-doped a-Si:C:H & $300 \mu \mathrm{m}$ & 40 & $\mathrm{Ar}-\mathrm{C}_{2} \mathrm{H}_{6}$ & $-1000 \mathrm{~V}$ \\
\hline
\end{tabular}

and cathodes and perturb the electric field or induce discharges.[13] GMDs have been found to be particularly prone to fast ageing possibly because of the smaller effective area used for charge multiplication on the anode strips or of a more effective polymerization process[14].

Two GMD devices, with and without p-doped a-Si:C:H coating on the anode-cathode strips, were irradiated by an X-ray tube at a rate of $1.8 \times 10^{5} \mathrm{~mm}^{-2} \mathrm{sec}^{-1}$. At fixed time intervals, the $\mathrm{X}$-ray tube was turned off and the detector was tested using an ${ }^{55} \mathrm{Fe}$ source. The ageing rate, $R$, is defined as

$$
\mathrm{R}=-\frac{1}{\mathrm{G}_{\mathrm{o}}} \frac{\mathrm{dG}}{\mathrm{dQ}} \text { in }[\% / \mathrm{C} / \mathrm{cm}]
$$

where $G_{o}$ is the initial gas gain, $G$ is the gas gain at a time $t$, and $Q$ is the collected charge per unit length of an anode strip for time $\mathrm{t}$. The detector overcoated with a-Si:C:H aged more slowly by approximately by a factor of 3 than the one without surface coating. Since the a-Si:H coats uniformly on the metal strips following the surface contour, it rounds off any sharp corners and edges. This rounding-off is believed to reduce accumulation of polymeric deposits and enhances ageing characteristics. Further study is in progress to clarify the effect of surface coatings on the ageing behavior.

\section{CONCLUSION}

Thin $(0.1 \sim 0.2 \mu \mathrm{m})$, p-type a-Si:C:H films have been applied to gas microstrip detectors to control surface resistivity. The surface resistivity was successfully controlled in the range of $10^{12}$ $\sim 10^{17} \Omega / \square$ and the light sensitivity was minimized by changing the relative amount of the carbon content and doping level in the film. The p-doped a-Si:C:H layers reached a steady-state leakage current value much faster than the undoped a-Si:H. When tested with ${ }^{55} \mathrm{Fe}$ source, maximum gas 
gains of $\sim 5000$, rate capabilities of $10^{5}$ counts $/ \mathrm{mm}^{2} \cdot \mathrm{sec}$, and energy resolution of $\leq 20 \%$ FWHM were achieved. The gain of the detector with a-Si:C:H coating $\left(70 \% \mathrm{CH}_{4}\right.$ and $\left.600 \mathrm{ppm} \mathrm{B}_{2} \mathrm{H}_{6}\right)$ remained constant over a week of operation. Performance of the detectors with a-Si:C:H layer was comparable to that with electronic-conducting glass film. Upon prolonged irradiation, the detector overcoated with a-Si:C:H aged more slowly by approximately by a factor of 3 than the one without surface coating. A boron-doped a-Si:C:H film is a good alternative to ion-implanted or semiconducting glass, or other conductive coatings which have been used, due to the ease with which large area may be covered, the wide range of surface resistivities possible, and the low cost of fabrication.

\section{ACKNOWLEDGMENT}

This work was supported by the Director, Office of Energy Research of the U.S. Department of Energy under Contract No. DE-AC03-76SF00098.

\section{REFERENCES}

[1] F.Angelini et al., Nucl. Instr. Meth., A 31521 (1992)

[2] F.Sauli, CERN/DRDC/93-34, RD-28 Status Report (1993)

[3] R.Bouclier et al., Nucl. Instr. Meth., A 332100 (1993)

[4] G.D.Minakov et al., Nucl. Instr. Meth., A 326566 (1991)

[5] A.Savard et al., Nucl. Instr. Meth., A337 387 (1993)

[6] Q.Wang, E.A.Schiff and Y.Li, Mat. Res. Soc. Symp. Proc., 297419 (1993)

[7] D.M.Bhusari et al., J. Non-Cryst. Sol., 137\&138 689 (1991)

[8] D.E.Anderson and W.E.Spear, Phil. Mag. B, 351 (1977)

[9] R.A.Street, Phil. Mag. B, 631343 (1991)

[10] R.A.Street, Appl. Phys. Lett., 571334 (1990)

[11] R.Bouclier et al., 1993 IEEE Conference Record, Vol.1 413 (1993)

[12] F.Angelini et al, Nucl. Instr. Meth., A 314450 (1992)

[13] J.Bohm et al, CERN-PPE/94-115, Presented at the 6th Pisa Conference, Elba, May 24-28, 1994

[14] R.Bouclier et al., CERN-PPE/94-63 Submitted to Nucl. Instr. Meth., (1994) 